\title{
In vivo Optical Coherence Tomography Imaging of the Mesothelium Using Developed Window Models
}

\author{
Yeh-Chan Ahn ${ }^{1,2 *}$, Yu-Gyeong Chae ${ }^{1,2} *$, Sang Seok Hwang ${ }^{1,2}$, \\ Bong-Kwon Chun ${ }^{2,3}$, Maan Hong Jung ${ }^{4}$, Sung Jin Nam${ }^{4}$, Hae-Young Lee ${ }^{5}$, \\ Jae Min Chung ${ }^{6}$, Chulho Oak ${ }^{2,4 \dagger}$, and Eun-Kee Park ${ }^{2,7} * *$ \\ ${ }^{I}$ Department of Biomedical Engineering and Center for Marine-Integrated Biomedical Technology, \\ Pukyong National University, Busan 608-737, Korea \\ ${ }^{2}$ Innovative Biomedical Technology Research Center, Busan 608-737, Korea \\ ${ }^{3}$ Department of Pathology, Kosin University College of Medicine, Busan 602-703, Korea \\ ${ }^{4}$ Department of Internal Medicine, Kosin University College of Medicine, Busan 602-703, Korea \\ ${ }^{5}$ Department of Chest Surgery, Kosin University College of Medicine, Busan 602-703, Korea \\ ${ }^{6}$ Department of Urology, Pusan National University Yangsan Hospital, Busan 626-870, Korea \\ ${ }^{7}$ Department of Medical Humanities and Social Medicine, Kosin University College of Medicine, \\ Busan 602-703, Korea
}

(Received December 9, 2014 : revised January 8, 2015 : accepted January 8, 2015)

\begin{abstract}
The mesothelium is an essential lining for maintaining the normal homeostasis of the closed body cavity and a central component of pathophysiologic processes. The mesothelium has been known as the end target for asbestos which induces asbestos-related lung diseases. Malignant mesothelioma (MM) is a rare and fatal neoplasm predominantly due to asbestos exposure. Adaptation of an advanced and reliable technology is necessary for early detection of MM because it is difficult to diagnose this disease in its early stages. Optical coherence tomography (OCT) provides cross-sectional images of micro-tissue structures with a resolution of 2-10 $\mu \mathrm{m}$ that can image the mesothelium with a thickness of $\sim 100 \mu \mathrm{m}$ and, therefore, enable investigation of early development of MM. The mesothelium is typically located at the pleura and tunica vaginalis of the scrotum. In this study, we developed animal window models in the above two anatomical sites to visualize mesothelial layers within the mesothelium. OCT images at the two locations were also acquired.
\end{abstract}

Keywords: Mesothelium, Optical coherence tomography, Pleura, Tunica vaginalis, Window model OCIS codes : (170.6935) Tissue characterization; (170.3880) Medical and biological imaging; (170.1610) Clinical applications; (170.4500) Optical coherence tomography

\section{INTRODUCTION}

Mesothelial proliferation, subsequent fibrosis, and mesothelioma are widely accepted complications of asbestos-related mesothelial injury [1]. The main target of asbestos fiber after exposure is the mesothelium, an essential lining for maintaining the normal homeostasis of the closed body cavity and a central component of pathophysiologic processes. The mesothelium is normally located at the pleura, pericardium, peritoneum, and tunica vaginalis (TV) of the scrotum (Fig. 1).
Malignant mesothelioma (MM) is a rare and fatal neoplasm which is predominantly caused by exposure to asbestos [2]. The age-adjusted mortality rate of MM has globally increased by $5.4 \%$ each year [3]. With regard to anatomical sites, the pleura accounts for $41.3 \%$ of all mesothelioma deaths and the peritoneum and pericardium for $4.5 \%$ and $0.3 \%$ of deaths, respectively [3]. Patients with MM have a median survival of less than 18 months $[4,5]$. MM is difficult to diagnose in its early stages which is the best stage to eradicate it; thus, a new concept of early diagnostic

\footnotetext{
*The authors equally contributed to this work.

*Corresponding authors: **ekpark@kosin.ac.kr, †oaks70@hanmail.net

Color versions of one or more of the figures in this paper are available online.
} 


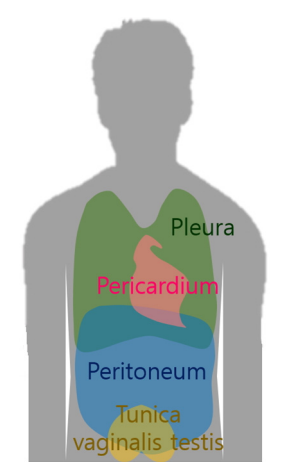

FIG. 1. The mesothelium is normally located at the pleura, pericardium, peritoneum, and tunica vaginalis of scrotum.

tools is required to achieve early diagnosis. Computed tomography $(\mathrm{CT})$ and positron emission tomography-computed tomography (PET-CT) are generally used to detect asbestosrelated diseases including MM. The diagnostic yield of CT and PET-CT, however, is not high because of limited resolution [6-10]. Moreover, it is not clear how asbestos causes mesothelioma and what are the diagnostic differences between mesothelioma and tuberculosis. The lack of knowledge requires basic studies using emerging imaging modalities and well-established animal models.

Recently, optical coherence tomography (OCT) has been developed which is an imaging modality with non-invasive, high-speed, high-resolution capabilities [11]. Even if OCT has a limited imaging depth, it provides real-time cross-sectional images with a micrometer scale. Several OCT studies related to pulmonology have been published [12]. The Brenner group demonstrated the first in vivo thoracoscopic imaging of pleural cancer using a three dimensional OCT system utilizing an improved forward-scanning rigid GRIN lens rod probe [13]. In vivo three dimensional imaging of the rabbit chest wall and lung can differentiate normal tissue and tumors on the chest. In their study, thoracoscopic surgery was performed on rabbits and the rod OCT probe was inserted into its pleural cavity. In order to image pleurae including lung parenchyma, however, we made a thoracic window [14]. It enabled us to utilize a bench-top OCT scanner with larger scanning area and better image quality than probe-based scanners. OCT endoscopic probes usually have a small field of view and a large f-number due to size limitation.

The thoracic window may provide direct visualization of both pleura but is difficult to develop and maintain because the thoracic window may cause severe complications such as pneumothorax. An alternative window for mesothelial visualization is in need more than ever. Besides pleural mesothelium, there is another kind of mesothelium in the scrotum that could be a potential window model accessed more easily than the pleura by OCT. Scrotal mesothelium called tunica vaginalis is also composed of visceral and parietal mesothelial layers and has anatomical similarity to the pleural mesothelium. Therefore, OCT may have a potential role in imaging mesothelial layers of the TV. However there has been no study on mesothelium of the TV using OCT. In this study, we investigated the feasibility of OCT for imaging pleural layers through a thoracic window and compared the result to OCT imaging of the mesothelial layers in the TV.

\section{METHODS}

\subsection{Thoracic Window Preparation}

All experiments were performed in accordance with the Guide for the Care and Use of Laboratory Animals (DHEW publication NIH 85-23, revised 2010, Office of Science and Health Reports, DRR/NIH, Bethesda, MD). The study was approved by the animal care and use committee of the College of Medicine at Kosin University. Male New Zealand white rabbits weighing 3.0 to $3.5 \mathrm{~kg}$ were used in this study. The initial intramuscular anesthesia was performed with ketamine $5-\mathrm{mg} / \mathrm{kg}$ and xylazine $0.8-\mathrm{mg} / \mathrm{kg}$. We maintained the anesthetic depth by injection of $10-\mathrm{mg} / \mathrm{h}$ ketamine and $3-\mathrm{mg} / \mathrm{h}$ xylazine. Oxygen saturation was monitored with a pulse oximeter in the ear. The rabbits were fixed on the operation table in a lateral position for preparation and image acquisition. The operating field was clipped and shaved. The skin was surgically opened with a 5-cm-long incision, and the upper intercostal muscle layers were resected between the third and fourth rib. The lower layers of muscle tissue were blunt dissected using small forceps, while all muscle fibers overlying the parietal pleura were removed in a region of approximately $10 \times 5 \mathrm{~mm}^{2}$. Resection of the lower muscle fibers had to be carried out very carefully to avoid injury of the pleura and lung tissue, pneumothorax, or mechanical stress during preparation.

\subsection{Tunica Vaginalis Window Preparation}

The rabbits were fixed on the operation table in a supine position for preparation and image acquisition. The operating field was clipped and shaved. The skin was surgically opened with a 2-cm-long incision and the upper scrotal fascia was removed. The lower layers of scrotal fasciae were blunt dissected using small forceps until the parietal tunica vaginalis was seen.

\subsection{OCT System}

A spectral-domain OCT system was developed based on the Michelson interferometer as shown in Fig. 2. A low coherence light source (BroadLighter D855, Superlum, Ireland) with a center wavelength of $850 \mathrm{~nm}$ and a FWHM of 100 $\mathrm{nm}$ was connected to the interferometer. The interference pattern was measured by a 1×4096 line scan camera (Sprint spL4096-140km, Basler, PA, USA) with a line rate of 140 $\mathrm{kHz}$. A two-axis scanner was designed and manufactured using two galvanometers $(6220 \mathrm{H}$, Cambridge Technology, MA, USA). The field of view was $10 \times 10 \mathrm{~mm}$. In order to characterize the OCT system, we measured the point spread 


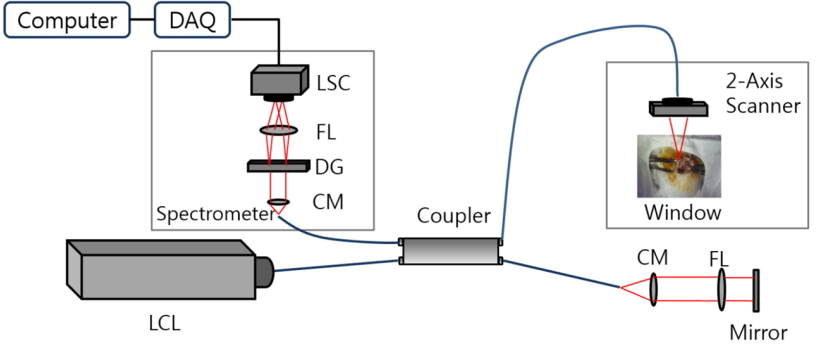

FIG. 2. Schematic diagram of the developed optical coherence tomography system. LSC: line scan camera, FL: focusing lens, DG: diffraction grating, CM: collimating lens, LCL: low coherence light, DAQ: data acquisition system.

function and it showed a spatial depth resolution of $3 \mu \mathrm{m}$ in air, a roll-off of $12 \mathrm{~dB} / \mathrm{mm}$, and a signal-to-noise ratio of $103 \mathrm{~dB}$. The lateral resolution was $13 \mu \mathrm{m}$. The frame rate was 70 frames per second with the image size of $1024 \times 2048$ pixels, which could minimize motion artifacts (70 times faster than rabbit's respiration rate).

\subsection{Histology}

The rabbits were euthanized using $\mathrm{CO}_{2}$ gas. Lung tissue (including pleura, alveoli, intercostal muscle, fascia, skin) and scrotal tissue (including tunica vaginalis, seminiferous tubules, muscle, fascia, skin) were cut into $6 \times 6 \mathrm{~cm}^{2}$ pieces, fixed by $10 \%$ neutral buffered formalin (NBF), and embedded in paraffin. Serial sections ( $4 \mu \mathrm{m}$ thick) were stained with hematoxylin and eosin (H\&E) and examined by microscopy.

\section{RESULTS AND DISCUSSION}

Figure 3(a) shows the thoracic window. Histology of the parietal layer (PL) and visceral layer (VL) with alveoli is shown in Figs. 3(b) and 3(c), respectively. OCT could visualize in vivo the PL, VL, pleural cavity between the PL and VL, and alveolar structure in real-time (Figs. 3(d) and 3(e)). The sliding movement of parietal and visceral pleurae according to respiration was also monitored by real-time OCT through the thoracic window.

Figure 3(f) is a photo of the TV window. Histology of the PL and VL in the TV is shown in Figs. 3(g) and 3(h). We could observe both the parietal mesothelium and visceral mesothelium of the tunica vaginalis using OCT (Figs. 3(i) and $3(\mathrm{j})$ ). We conducted three-dimensional OCT imaging and visualized the detailed structures in the scrotum and testis using OCT (Fig. 4). A total of 512 cross-sectional OCT images were volume-rendered (the number of voxels was $1024 \times 2048 \times 512$ and the size of each voxel was $5 \times 3 \times 5$ $\mu \mathrm{m}^{3}$ ) and en face images were reconstructed as shown in Fig. 4(a). It shows multiple seminiferous tubules and testicular septa with the PL and VL.

MM is an incurable cancer with an increasing incidence worldwide. Recent studies have focused on molecular

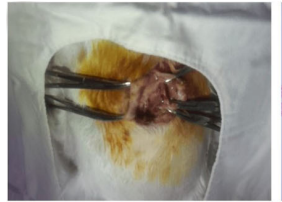

(a)

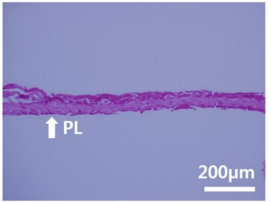

(b)

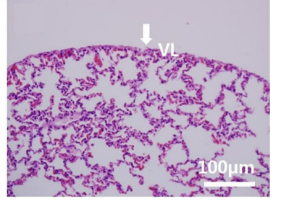

(c)

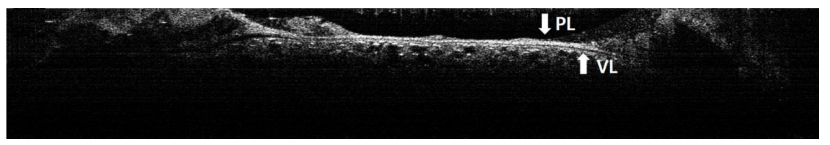

(d)

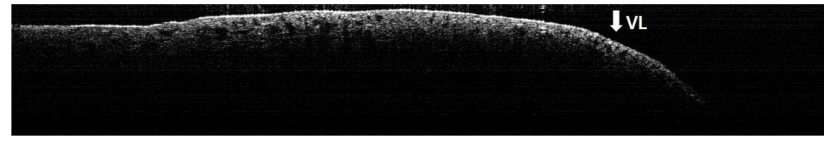

(e)

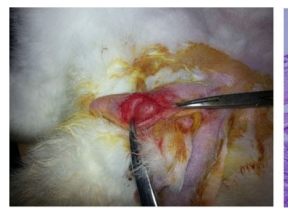

(f)

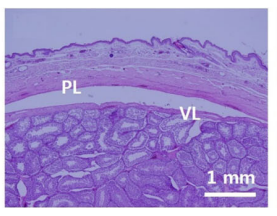

(g)

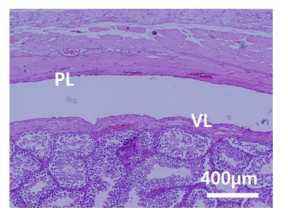

(h)

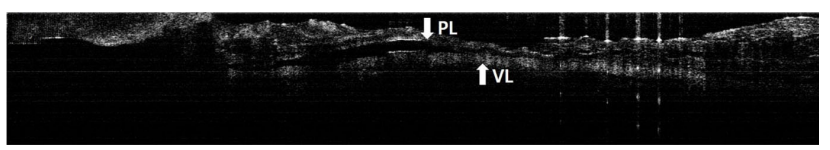

(i)

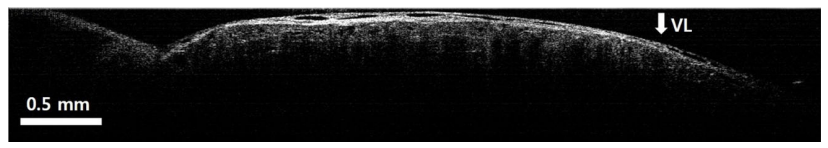

(j)

FIG. 3. Two window models developed in this study. (a) Thoracic window model, (b) Histology of parietal pleura, (c) Histology of visceral pleura and lung parenchyma, (d) OCT image of parietal and visceral pleurae and pleural cavity in between, (e) OCT image of visceral pleura and lung parenchyma, (f) Tunica vaginalis (TV) window model, (g-h) Histology of TV with parietal and visceral layers, (i) OCT image of parietal and visceral layers of TV (Saline was injected between two layers to discriminate them easily), (j) OCT image of visceral layer of TV. PL: parietal layer, VL: visceral layer.

approaches for early detection of MM $[15,16]$. However, sensitivity and specificity criteria of biomarker usage need to be improved. In order to develop strategic MM early detection methods, application of new technology is required. Basic research with OCT and animal window models could provide effective early detection methods. Although the pleura is the main site of mesothelioma incidence, to better understand mesothelioma other sites including the mesothelium with easy accessibility should be beneficial in regard to early diagnosis and monitoring of disease progression. An alternative mesothelium with the potential for studying mesothelial disease is located in the TV, but this area has 


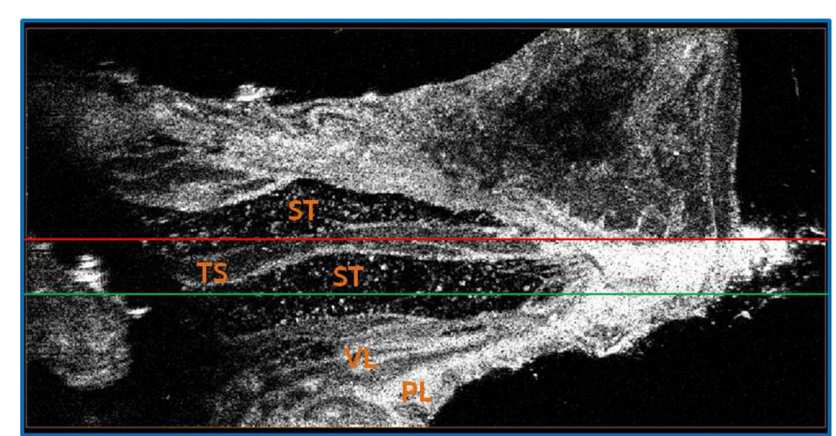

(a)

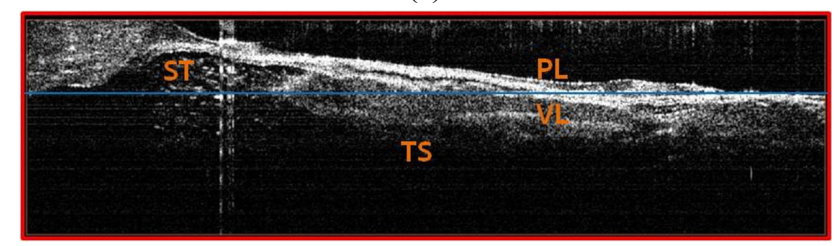

(b)

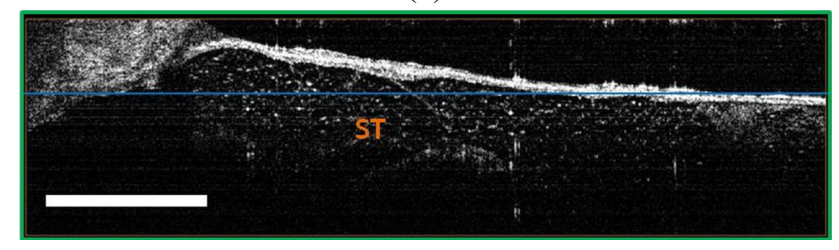

(c)

FIG. 4. Three dimensional OCT imaged the detailed structure in rabbit scrotum through the developed tunica vaginalis window. (a) En face OCT image, (b) Cross-sectional image along the red line on (a), (c) Cross-sectional image along the green line on (a). TS: Testicular Septa, PL: parietal layer, VL: visceral layer, ST: seminiferous tubule. Scale bar: $1 \mathrm{~mm}$.

received little attention by researchers thus far. However, it is clinically a well-known site for tuberculosis and MM $[17,18]$. Moreover, the TV provides easy accessibility and its anatomical structure is similar to the pleura. Therefore, the TV model can potentially give us the advantage of further studies such as the development of tumors or tuberculosis in the mesothelium. The thoracic and TV windows could let us visualize mesothelial layers synergistically and showed the potential for OCT to be an early diagnostic tool for mesothelial disease within the mesothelium. In this study, OCT could more easily visualize mesothelial layers in the TV than those of the pleura. In a previous study, a rabbit pleural tumor model was successfully developed [19]. Thus, in the near future, a rabbit TV tumor model will be a promising model of mesothelioma. OCT will be a potential tool for in vivo monitoring of tumorigenesis in the TV with time intervals.

The structural changes of the mesothelium according to asbestos exposure include diffuse pleural thickening (DPT), pleural plaques, and MM [20]. Among the pleural changes, DPT and pleural plaques could be the end products from either tuberculosis or asbestos. The differential diagnosis of DPT and pleural plaques from a diagnostic view has become important. In addition, an asbestos compensation law has also been established [21]. Therefore, the TV window model with an advanced diagnostic tool to differentiate MM from tuberculosis needs to be further investigated.

Currently, it is not clear how asbestos causes mesothelioma and what are the diagnostic differences between mesothelioma and tuberculosis. The lack of knowledge requires basic studies using emerging imaging modalities such as OCT and well-established animal models. OCT showed a fast imaging speed (70 fps) and a high spatial resolution (3 $\mu \mathrm{m})$ which were enough to minimize motional artifacts and measure the thickness of pleura. However, OCT has a limit in imaging depth. Therefore, clinical study can be tried with multimodal imaging modalities such as high resolution $\mathrm{CT}$ and photoacoustic tomography after the basic studies on the onset mechanism and progression of malignant mesothelioma are conducted.

\section{CONCLUSION}

The two window models for visualizing the mesothelium using OCT were feasible. Compared with the thoracic window, the tunica vaginalis model provided better accessibility and reliability than the thoracic window model. The house-made optical coherence tomography system was utilized to image mesothelial layers in the pleura and tunica vaginalis in vivo using two window models. The three-dimensional micro-structure of the tunica vaginalis and testis was also imaged. The images confirmed that mesothelial layers in the tunica vaginalis were similar to those in the pleura. The tunica vaginalis window model may benefit the development of a diagnostic tool to differentiate between mesothelioma and tuberculosis.

\section{ACKNOWLEDGMENT}

This study was supported by the National Research Foundation of Korea (2013R1A1A4A01011631, 2013K1A3A1A09076144, 2011-0006286).

\section{REFERENCES}

1. B. W. Robinson, A. W. Musk, and R. A. Lake, "Malignant mesothelioma," Lancet 366, 397-408 (2005).

2. J. Peto, A. Decarli, C. La Vecchia, F. Levi, and E. Negri, "The European mesothelioma epidemic," Br. J. Cancer 79, 666-672 (1999).

3. V. Delgermaa, K. Takahashi, E. K. Park, G. V. Le, T. Hara, and T. Sorahan, "Global mesothelioma deaths reported to the World Health Organization between 1994 and 2008," Bull. World Health Organ. 89, 716-724, 724A-724C (2011).

4. R. A. Hyland, S. Ware, A. R. Johnson, and D. H. Yates, "Incidence trends and gender difference in malignant 
mesothelioma in New South Wales, Australia," Scand. J. Work Environ. Health 33, 286-292 (2007).

5. D. Martino and H. I. Pass, "Integration of multimodality approached in the management of malignant mesothelioma," Clin. Lung Cancer 5, 290-298 (2004).

6. B. W. Robinson and R. A. Lake, "Advances in malignant mesothelioma," N. Engl. J. Med. 353, 1591-1603 (2005).

7. T. Saraya, T. Yokoyama, H. Ishii, Y. Tanaka, N. Tsujimoto, Y. Ogawa, E. Sohara, A. Nakajima, T. Inui, H. Sayuki, M. Fujiwara, T. Oka, R. Kawachi, T. Goya, H. Takizawa, and H. Goto, "A case of malignant peritoneal mesothelioma revealed with limitation of PET-CT in the diagnosis of thoracic metastasis," J. Thorac. Dis. 5, E11-16 (2013).

8. K. Kawaguchi, T. Taniguchi, N. Usami, T. Fukui, F. Ishiguro, S. Nakamura, and K. Yokoi, "FDG PET/CT is useful for detecting infiltration to the port site in patients with malignant pleural mesothelioma," Gen. Thorac. Cardiovasc. Surg. 62, 157-162 (2014).

9. M. T. Truong, C. Viswanathan, M. B. Godoy, B. W. Carter, and E. M. Marom, "Malignant pleural mesothelioma: Role of CT, MRI, and PET/CT in staging evaluation and treatment considerations," Semin. Roentgenol. 48, 323-334 (2013).

10. B. Sureka, B. B. Thukral, M. K. Mittal, A. Mittal, and M. Sinha, "Radiological review of pleural tumors," Indian J. Radiol. Imaging 23, 313-320 (2013).

11. D. Huang, E. A. Swanson, C. P. Lin, J. S. Schuman, W. G. Stinson, W. Chang, M. R. Hee, T. Flotte, K. Gregory, and C. A. Puliafito, "Optical coherence tomography," Science 254, 1178 (1991).

12. R. Hou, T. Le, S. D. Murgu, Z. Chen, and M. Brenner, "Recent advances in optical coherence tomography for the diagnoses of lung disorders," Expert Rev. Respir. Med. 5, 711-724 (2011).

13. T. Xie, G. Liu, K. Kreuter, S. Mahon, H. Colt, D. Mukai, G. M. Peavy, Z. Chen, and M. Brenner, "In vivo threedimensional imaging of normal tissue and tumors in the rabbit pleural cavity using endoscopic swept source optical coherence tomography with thoracoscopic guidance," J. Biomed. Opt. 14, 064045 (2009).

14. S. Meissner, L. Knels, C. Schnabel, T. Koch, and E. Koch, "Three-dimensional Fourier domain optical coherence tomography in vivo imaging of alveolar tissue in the intact thorax using the parietal pleura as a window," J. Biomed. Opt. 15, 016030 (2010).

15. H. I. Pass, S. M. Levin, M. R. Harbut, J. Melamed, L. Chiriboga, J. Donington, M. Huflejt, M. Carbone, D. Chia, L. Goodglick, G. E. Goodman, M. D. Thornquist, G. Liu, M. de Perrot, M. S. Tsao, and C. Goparaju, "Fibulin-3 as a blood and effusion biomarker for pleural mesothelioma," N. Engl. J. Med. 367, 1417-1427 (2012).

16. M. B. Kirschner, Y. Y. Cheng, B. Badrian, S. C. Kao, J. Creaney, J. J. Edelman, N. J. Armstrong, M. P. Vallely, A. W. Musk, B. W. Robinson, B. C. McCaughan, S. Klebe, S. E. Mutsaers, N. van Zandwijk, and G. Reid, "Increased circulating miR-625-3p: A potential biomarker for patients with malignant pleural mesothelioma," J. Thorac. Oncol. 7, 1184-1191 (2012).

17. P. Sookpotarom, K. Nimanussornkul, O. Luecha, R. Poolsavatkitikool, and P. Vejchapipat, "Isolated tuberculosis of tunica vaginalis in a child," Pediatr. Surg. Int. 26, 763-765 (2010).

18. S. Erdogan, A. Acikalin, H. Zeren, G. Gonlusen, S. Zorludemir, and V. Izol, "Well-differentiated papillary mesothelioma of the tunica vaginalis: A case study and review of the literature," Korean J. Pathol. 48, 225-228 (2014).

19. K. A. Kreuter, N. El-Abbadi, A. Shbeeb, L. Tseng, S. B. Mahon, N. Narula, T. Burney, H. Colt, and M. Brenner, "Development of a rabbit pleural cancer model by using VX2 tumors," Comp. Med. 58, 287-293 (2008).

20. M. Bayram and N. D. Bakan, "Environmental exposure to asbestos: From geology to mesothelioma," Curr. Opin. Pulm. Med. 20, 301-307 (2014).

21. N. Ndlovu, J. T. Naude, and J. Murray, "Compensation for environmental asbestos-related diseases in South Africa: A neglected issue," Glob. Health Action 6, 19410 (2013). 